\title{
High-precision masses of neutron-deficient rubidium isotopes using a Penning trap mass spectrometer
}

\author{
A. Kellerbauer,,${ }^{1, *}$ G. Audi, ${ }^{2}$ D. Beck,${ }^{3}$ K. Blaum,${ }^{3,4}$ G. Bollen, ${ }^{5}$ C. Guénaut,,${ }^{2, \dagger}$ F. Herfurth, ${ }^{3}$ A. Herlert,,${ }^{6, \ddagger}$ \\ H.-J. Kluge, ${ }^{3}$ D. Lunney, ${ }^{2}$ S. Schwarz, ${ }^{5}$ L. Schweikhard, ${ }^{6}$ C. Weber,,${ }^{3,8}$ and C. Yazidjian ${ }^{3}$ \\ ${ }^{1}$ Physics Department, CERN, CH-1211 Genève 23, Switzerland \\ ${ }^{2}$ CSNSM-IN2P3-CNRS, F-91405 Orsay-Campus, France \\ ${ }^{3}$ GSI, Planckstraße 1, D-64291 Darmstadt, Germany \\ ${ }^{4}$ Institut für Physik, Johannes-Gutenberg-Universität, D-55099 Mainz, Germany \\ ${ }^{5}$ NSCL, Michigan State University, East Lansing, Michigan 48824-1321, USA \\ ${ }^{6}$ Institut für Physik, Ernst-Moritz-Arndt-Universität, D-17487 Greifswald, Germany
}

(Received 30 April 2007; published 30 October 2007)

\begin{abstract}
The atomic masses of the neutron-deficient radioactive rubidium isotopes ${ }^{74-77,79,80,83} \mathrm{Rb}$ have been measured with the Penning trap mass spectrometer ISOLTRAP. Using the time-of-flight cyclotron resonance technique, relative mass uncertainties ranging from $1.6 \times 10^{-8}$ to $5.6 \times 10^{-8}$ were achieved. In all cases, the mass precision was significantly improved as compared with the prior Atomic-Mass Evaluation; no significant deviations from the literature values were observed. The exotic nuclide ${ }^{74} \mathrm{Rb}$, with a half-life of only $65 \mathrm{~ms}$, is the shortest-lived nuclide on which a high-precision mass measurement in a Penning trap has been carried out. The significance of these measurements for a check of the conserved-vector-current hypothesis of the weak interaction and the unitarity of the Cabibbo-Kobayashi-Maskawa matrix is discussed.
\end{abstract}

DOI: 10.1103/PhysRevC.76.045504

PACS number(s): 21.10.Dr, 07.75.+h, 27.50.+e, 32.10.Bi

\section{INTRODUCTION}

Via the equivalence of mass and energy, high-precision mass measurements give direct access to information on nuclear binding energies. Data from short-lived nuclides are of importance for a wide range of research subjects, from nuclear physics to astrophysics and the fundamental interactions between elementary particles [1,2]. Over the past decade, atomic-mass measurements at a relative precision of $10^{-7}$ or better have begun to make significant contributions to experimental tests of the conserved-vector-current (CVC) hypothesis of the weak interaction.

ISOLTRAP is a Penning trap mass spectrometer dedicated to high-precision mass measurements on exotic nuclides. It has been in operation since the second half of the 1980s and has already been used to carry out mass measurements on more than 300 radionuclides [3,4]. Over the past few years, the efficiency and precision of ISOLTRAP have been significantly improved [5], thus making the experiment applicable for highprecision measurements on very short-lived nuclides as those required for this study.

The CVC postulate was proposed by Feynman and Gell-Mann in 1958 [6] and is now an integral part of the

\footnotetext{
*Corresponding author; present address: Max Planck Institute for Nuclear Physics, P.O. Box 103980, D-69029 Heidelberg, Germany; a.kellerbauer@mpi-hd.mpg.de

${ }^{\dagger}$ Present address: NSCL, Michigan State University, East Lansing, MI 48824-1321, USA.

${ }^{\ddagger}$ Present address: Physics Department, CERN, CH-1211 Genève 23, Switzerland.

${ }^{\S}$ Present address: Department of Physics, University of Jyväskylä, P.O. Box 35 (YFL), FIN-40014 Jyväskylä, Finland.
}

Standard Model of particle physics. It states that the vector part of the weak interaction is not influenced by the strong force. The comparative half-life $f t$ of superallowed $\beta$ transitions between analog $J^{\pi}=0^{+} \longrightarrow 0^{+}$states (i.e., pure Fermi decays) should only be a function of the matrix element that connects the two states, which for decays between nuclides with isospin $T=1$ is simply $\left\langle M_{\mathrm{V}}\right\rangle^{2}=T(T+1)=2$, and the vector coupling constant $G_{\mathrm{V}}$ [7]. Therefore, the $f t$ values for all superallowed $\beta$ decays are expected to be exactly equal, aside from small corrections owing to the influence of the nuclear environment in which the decay takes place. The corrected $f t$ value $F t$ is defined as [7,8]

$$
F t \equiv f t\left(1+\delta_{R}\right)\left(1-\delta_{C}\right),
$$

where $\delta_{R}$ is the nucleus-dependent radiative correction and $\delta_{C}$ is the isospin-symmetry-breaking correction [8].

The Cabibbo-Kobayashi-Maskawa (CKM) matrix relates the weak-interaction eigenstates of the quarks to their mass eigenstates. Its first element $V_{u d}$ is determined by the simple relation

$$
V_{u d}^{2}=\frac{G_{V}^{2}}{G_{F}^{2}},
$$

where $G_{F}$ is the fundamental weak coupling constant, which can be determined from the purely leptonic muon decay. We can obtain $G_{V}$ from the mean $F t$ value of all superallowed $\beta$ decays, assuming the comparative half-lives are found to agree with each other, via the expression

$$
G_{V}^{2}=\frac{K}{2 \overline{F t}\left(1+\Delta_{R}^{V}\right)},
$$

where $K=2 \pi^{3} \hbar(\hbar c)^{6} \ln 2 /\left(m_{e} c^{2}\right)^{5}$ is a product of fundamental constants and $\Delta_{R}^{V}$ is a nucleus-independent radiative 
correction. Together with $V_{u s}$ and $V_{u b}$, the two other elements of the first row, $V_{u d}$ can be used to perform a partial unitarity test of the CKM matrix by checking whether the sum of the squares of these three elements is equal to unity.

Experimentally, Ft is accessible via the following measured quantities: the decay energy $Q$, which enters to the fifth power into the calculation of the statistical rate function $f$ [9], the half-life $T_{1 / 2}$, and the branching ratio $R$. The latter two yield the partial half-life $t=T_{1 / 2}\left(1+P_{\mathrm{EC}}\right) / R$, where $P_{\mathrm{EC}}$ is the calculated electron capture fraction. Precise $F t$ values with relative uncertainties better than $10^{-4}$ are known for nine superallowed decays with $A \leqslant 54$, and they generally agree well with each other and their mean value $\overline{F t}=$ 3073.5(1.2) s [8].

Prior to the measurements reported here, the atomic mass of ${ }^{74} \mathrm{Rb}$, and thus the decay energy of its superallowed $\beta$ decay, was only known to $720 \mathrm{keV}$, thus making a calculation of $F t$ for that decay meaningless. To extend the base of available data toward heavier nuclides in a new nuclear-shell region, we have performed mass measurements on ${ }^{74} \mathrm{Rb}$, as well as other neutron-deficient rubidium isotopes, with the ISOLTRAP experiment installed at CERN in Geneva, Switzerland. The masses of the $\beta$-decay daughter ${ }^{74} \mathrm{Kr}$ and other krypton isotopes were also measured with ISOLTRAP during another set of data-taking periods, and those results have already been published [10].

In addition to the special case of ${ }^{74} \mathrm{Rb}$, high-precision mass measurements are of great interest for nuclear structure and for constraining nuclear (mass) models. For example, the study of the residual neutron-proton interaction [11,12] involves the second derivative of the mass surface, which requires data of good precision to elaborate the trends and hence elucidate the structure. Sometimes, mass data are improved not only by reducing the uncertainty but in revealing errors that misdirect extrapolation efforts. This is important in the development of mass models, not only for correcting wrong tendencies for extrapolation but also for providing reliable data with which the range of parameter variation can be adjusted [1].

We have reported on the mass excess of ${ }^{74} \mathrm{Rb}$ as well as the decay energy of its superallowed $\beta$ decay in a recent Letter [13]. In the present article, we present the mass results for all studied rubidium isotopes and the other nuclides whose masses were also measured in the course of the same datataking periods. Our masses are discussed in comparison with prior measurements, and the result of an updated Atomic-Mass Evaluation incorporating the new masses is presented.

\section{SETUP AND TECHNIQUE}

At CERN's ISOLDE facility [14], radioactive nuclides are produced by bombarding a thick target with high-intensity proton pulses from the Proton Synchrotron Booster at energies up to $1.4 \mathrm{GeV}$. Spallation, fragmentation, and fission reactions in the target produce a wide range of nuclides, both stable and radioactive, which diffuse out of the heated target material, through a transfer line and toward an ion source. The ionized samples are then accelerated to $60-\mathrm{keV}$ kinetic energy and mass-separated in one of ISOLDE's magnetic separators. The
General-Purpose Separator (GPS) and the High-Resolution Separator (HRS) are equipped with separate target stations and operate at resolving powers of about 1500 and about 3000 , respectively. Together with the chemical selectivity afforded by the use of different line temperatures and ion source types, a high degree of selectivity is thus obtained. Downstream of the separators, the mass-separated $60-\mathrm{keV}$ ion beams are merged into the main beamline and distributed to any of the experiments installed at the ISOLDE experimental hall.

ISOLTRAP is located at the end of ISOLDE's central beamline. It consists of three ion traps, which serve the distinct purposes of decelerating and bunching and cooling the ISOLDE ion beam, removing isobaric contaminants, and performing the actual mass measurement. The detailed experimental setup is described elsewhere [15]. For the purpose of this article, we will limit ourselves to a brief review of the time-of-flight (TOF) resonance technique [16] as applied at ISOLTRAP [17] for high-precision mass measurements.

An ion confined in a Penning trap performs the superposition of three simple harmonic modes, one axial and two radial [18]. The radial motion consists of a slow magnetron motion at frequency $f_{-}$and a high-frequency cyclotron motion at the modified frequency $f_{+}$. In an ideal Penning trap, the sum of the radial frequencies is exactly the cyclotron frequency $f_{c}$ of an ion in a purely magnetic field:

$$
f_{+}+f_{-}=f_{c}=\frac{1}{2 \pi} \frac{q B}{m},
$$

where $q$ and $m$ are the charge and mass of the ion and $B$ is the magnitude of the magnetic field. When the radial motions are resonantly excited at the sum frequency by means of an azimuthal quadrupolar radio-frequency (RF) signal, as shown in Fig. 1(a), they are continuously converted from one to the other. The conversion proceeds in the form of Rabi oscillations whose period is inversely proportional to the amplitude of the applied RF [17].

The mass measurement is carried out in ISOLTRAP's precision trap, a hyperbolical Penning trap located in the field of a 5.9-T superconducting magnet. When a single ion or a small ensemble of ions is loaded into the trap, they initially have very small magnetron and cyclotron orbits. The ions' magnetron motion is first increased to a well-defined amplitude by an azimuthal dipolar field at the magnetron frequency [19]. The radial motions are then excited by a quadrupolar field near the cyclotron frequency whose coupling strength (the product of field amplitude $U_{\text {exc }}$ and duration $T_{\text {exc }}$ ) is carefully chosen such that only one full conversion of magnetron into cyclotron motion takes place.

Subsequently, the resonant excitation of the ions is detected. For this purpose, the ions are ejected out of the trap and onto a particle detector about $1.4 \mathrm{~m}$ downstream, outside the high-magnetic-field region, as shown in Fig. 1(b). In the inhomogeneous magnetic field between the trap center and the detector, the ions experience an axial accelerating force owing to their orbital magnetic moment. Since that force is proportional to the radial energy of the ions prior to ejection, the TOF is a measure of the degree to which the low-energy magnetron motion has been converted into cyclotron motion. A plot of the mean TOF as a function 


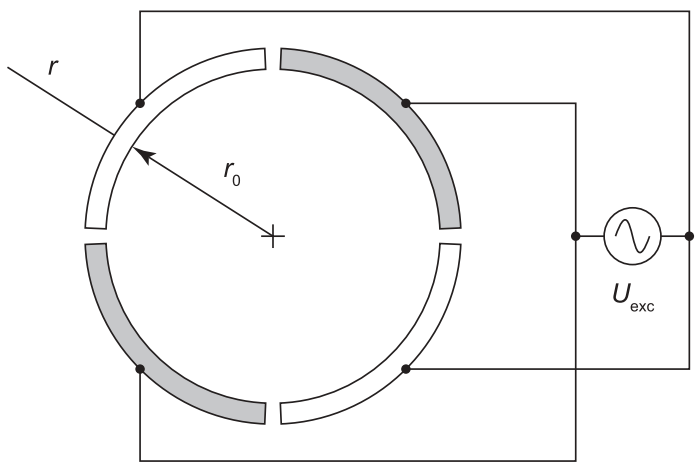

(a)

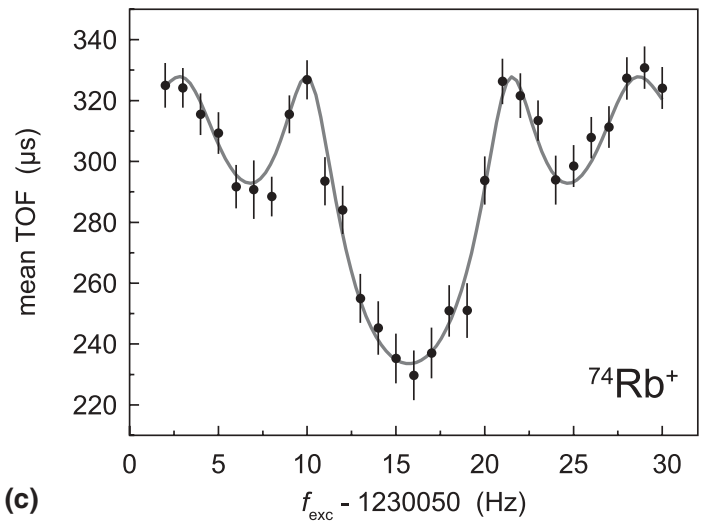

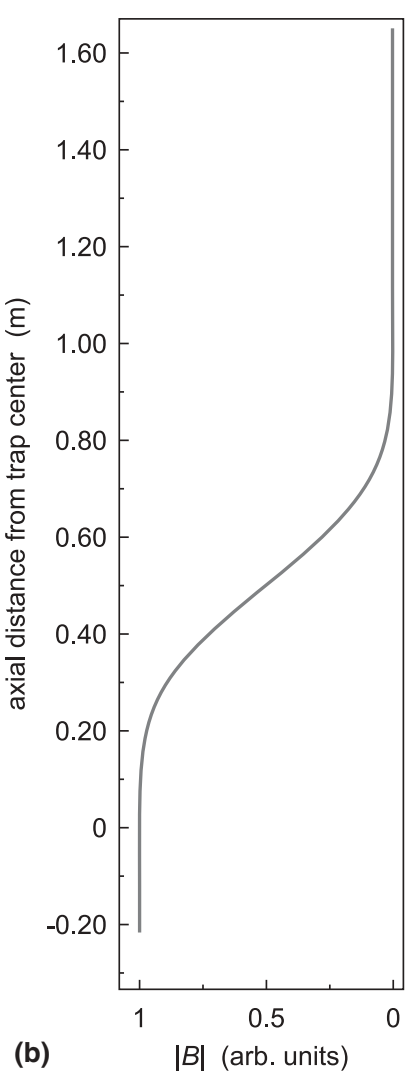

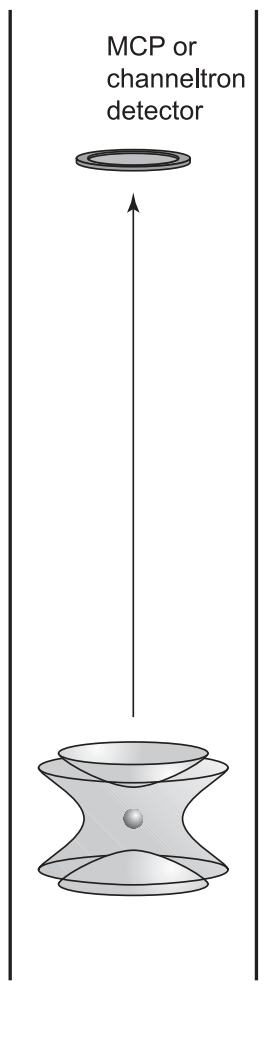

FIG. 1. Schematic of the TOF cyclotron resonance technique. (a) Azimuthal quadrupolar RF field applied via a fourfold segmented ring electrode of the Penning trap. (b) Upon ejection from the trap, the magnetic-field gradient exerts an axial accelerating force on the ions. (c) TOF cyclotron resonance spectrum for singly charged ${ }^{74} \mathrm{Rb}$ ions. The central TOF minimum corresponds to the cyclotron frequency of the ion.

of the excitation frequency for a substantial number of ions reveals a characteristic line shape that is fully understood theoretically [17]. A fit of the theoretical line shape to the data then yields the cyclotron frequency. Figure 1(c) shows a typical TOF cyclotron resonance spectrum for ${ }^{74} \mathrm{Rb}^{+}$.

To determine the mass of the ion from the cyclotron frequency by virtue of Eq. (4), the magnitude of the magnetic field $B$ must also be known to high precision. This is achieved by measuring the cyclotron frequency of a reference ion, whose mass is very well known, both before and after the unknown ion. Assuming that all ion species have the same charge $q=+e$, one can then calculate the atomic mass $m$ of the ion of interest from the cyclotron frequency of the reference ion, $f_{c \text {, ref }}$, the cyclotron frequency of the ion of interest, $f_{c}$, the atomic mass of the reference ion, $m_{\mathrm{ref}}$, and the electron mass $m_{e}$ :

$$
m=\frac{f_{c, \mathrm{ref}}}{f_{c}}\left(m_{\mathrm{ref}}-m_{e}\right)+m_{e} .
$$

The mass-resolving power $R=m / \delta m$ that can be achieved with the TOF cyclotron technique is directly proportional to the cyclotron frequency and the duration of the quadrupolar excitation [5]:

$$
R \approx f_{c} T_{\mathrm{exc}}
$$

With an observation time of $1 \mathrm{~s}$, a resolving power of $10^{6}$ can typically be reached for medium-heavy ions. Depending on the number of recorded events, relative statistical uncertainties of $10^{-8}$ to $10^{-9}$ are achievable. A recent comprehensive study using carbon cluster ions ${ }^{12} \mathrm{C}_{n}^{+}$revealed that ISOLTRAP's uncertainty limit is currently $8 \times 10^{-9}$ [20].

\section{EXPERIMENT}

The data presented here were collected during three distinct data-taking periods in 2000, 2002, and 2003 that lasted about one week each. The neutron-deficient rubidium isotopes were produced from niobium foil targets in conjunction with tungsten surface ionization ion sources. The targets were bombarded with proton pulses containing up to $3 \times 10^{13}$ protons each at kinetic energies of 1.0 or $1.4 \mathrm{GeV}$. The HRS, which was used in all of the measurements discussed here, was operated at a mass-resolving power of $m / \delta m \approx 3000$.

Except for ${ }^{74} \mathrm{Rb}$, all nuclides under investigation have halflives longer than $15 \mathrm{~s}$. They were measured using RF excitation times in the precision Penning trap of 300 to $1800 \mathrm{~ms}$. In the special case of ${ }^{74} \mathrm{Rb}$ with a half-life of only $65 \mathrm{~ms}$, a very short measurement cycle, such as that already used for ${ }^{32} \mathrm{Ar}$ [21], had to be employed. In that scheme, the total cycle time from the injection of the ions into the RFQ ion beam 
cooler and buncher to the mass measurement takes less than $200 \mathrm{~ms}$. The duration of the RF excitation was varied between 60 and $120 \mathrm{~ms}$, corresponding to mass-resolving powers of $m / \delta m \approx 80000$ to 160000 . Because of the extremely low production yield of ${ }^{74} \mathrm{Rb}$ of only a few hundred ions per proton pulse, a total measurement time of more than $54 \mathrm{~h}$ was required to record 10000 ions of that isotope alone. A typical TOF cyclotron resonance of ${ }^{74} \mathrm{Rb}$ is shown in Fig. 1(c). For the results reported in this article, a total of 63 resonances were taken, out of which 24 were for ${ }^{74} \mathrm{Rb}$. The data were carefully analyzed with respect to systematic and statistical uncertainties by following the data analysis procedure described in Ref. [20].

The reference measurements were carried out with the stable nuclide ${ }^{85} \mathrm{Rb}$ produced in ISOLTRAP's reference ion source. An RF excitation time of $900 \mathrm{~ms}$ was chosen, yielding a mass-resolving power of about $10^{6}$. References were obtained between all cyclotron frequency determinations of the ions of interest, thereby minimizing the uncertainty resulting from magnetic-field fluctuations, and the intervals between most references were kept below $3 \mathrm{~h}$ (below $6 \mathrm{~h}$ for the ${ }^{74} \mathrm{Rb}$ measurements).

To exclude systematic shifts caused by unknown sources, the masses of the stable nuclides ${ }^{74} \mathrm{Ga}$ and ${ }^{88} \mathrm{Sr}$ (from ISOLDE) as well as ${ }^{133} \mathrm{Cs}$ and ${ }^{87} \mathrm{Rb}$ (from the reference ion source), whose masses are well known, were measured in addition to the exotic nuclides. As discussed in detail in the following, they were found to be in good agreement with the literature values. In the course of investigating isobars at $A=74$, the mass of ${ }^{74} \mathrm{Ga}$ was also measured and its uncertainty reduced by a factor of 3 . It has since been superseded by a yet more precise ISOLTRAP datum [22].

All cyclotron frequency ratios of the three data-taking periods are given in Table I. The half-lives of the nuclides (from NUBASE [23]) are indicated in the second column; the measured cyclotron frequency ratios are shown in the third column. From the primary result of a Penning trap mass measurement (the cyclotron frequency ratio $r$ ), the atomic mass $m$ of the studied nuclide can be calculated according to Eq. (5) by using the currently best available values for the masses of the reference atom, $m_{\text {ref }}$, and the mass of the electron, $m_{e}$. The values that were used in the calculation of the mass results presented in the table are $M\left({ }^{85} \mathrm{Rb}\right)=84.911789738(12) \mathrm{u}$, $m_{e}=510.998902(21) \mathrm{keV}$, and $1 \mathrm{u}=931494.009$ (7) $\mathrm{keV}$ [25]. (The unit $\mathrm{V}$ used here is the standard volt rather than the international volt. For a justification, see Ref. [25].) The obtained masses and their relative uncertainties are shown in the fourth and fifth columns of Table I. An overview of the difference between mass measurements presented here and the

TABLE I. Half-lives $T_{1 / 2}$ (from NUBASE [23]) and measured cyclotron frequency ratios $r=v_{c}$,ref $/ v_{\mathrm{c}}$ for the nuclides measured during data-taking periods in 2000 (first set), 2002 (second set), and 2003 (third set). In all cases, ${ }^{85} \mathrm{Rb}$ was used as the reference mass. The experimental masses $m$ from the ISOLTRAP measurements and their relative uncertainties were calculated according to Eq. (5) using current values for the mass of ${ }^{85} \mathrm{Rb}$, the electron mass, and the unified atomic mass unit, all given in the text. Uncertainties (in parentheses) refer to the least significant digits of a quantity. The uncertainties of the frequency ratios are given to three figures to reduce rounding errors in subsequent calculations such as the Atomic-Mass Evaluation.

\begin{tabular}{lcccc}
\hline \hline Nuclide & $T_{1 / 2}$ & \multicolumn{1}{c}{$r$} & $m(\mathrm{u})$ & $u(m) / m$ \\
\hline${ }^{74} \mathrm{Ga}^{\mathrm{a}}$ & $8.12 \mathrm{~min}$ & $0.870631490(265)$ & $73.926949(22)$ & $3.0 \times 10^{-7}$ \\
${ }^{74} \mathrm{Rb}^{\mathrm{b}}$ & $64.8 \mathrm{~ms}$ & $0.870835571(226)$ & $73.944278(19)$ & $2.6 \times 10^{-7}$ \\
${ }^{76} \mathrm{Rb}^{\mathrm{b}}$ & $36.5 \mathrm{~s}$ & $0.8942811649(232)$ & $75.9350722(20)$ & $2.6 \times 10^{-8}$ \\
${ }^{88} \mathrm{Sr}$ & stable & $1.035258054(233)$ & $87.905595(20)$ & $2.2 \times 10^{-7}$ \\
${ }^{133} \mathrm{Cs}$ & stable & $1.5652215168(190)$ & $132.9054503(16)$ & $1.2 \times 10^{-8}$ \\
${ }^{64} \mathrm{Zn}$ & stable & $0.7528873458(984)$ & $63.9291476(84)$ & $1.3 \times 10^{-7}$ \\
${ }^{71} \mathrm{Ga}$ & stable & $0.8352740429(156)$ & $70.9247043(13)$ & $1.9 \times 10^{-8}$ \\
${ }^{74} \mathrm{Rb}$ & $64.8 \mathrm{~ms}$ & $0.8708354150(608)$ & $73.9442645(52)$ & $7.0 \times 10^{-8}$ \\
${ }^{75} \mathrm{Rb}$ & $19.0 \mathrm{~s}$ & $0.8825453928(199)$ & $74.9385733(17)$ & $2.2 \times 10^{-8}$ \\
${ }^{76} \mathrm{Rb}$ & $36.5 \mathrm{~s}$ & $0.894281062(176)$ & $75.935063(15)$ & $2.0 \times 10^{-7}$ \\
${ }^{87} \mathrm{Rb}$ & stable & $1.02352327679(943)$ & $86.90918037(80)$ & $9.2 \times 10^{-9}$ \\
${ }^{74} \mathrm{Ga}$ & $8.12 \mathrm{~min}$ & $0.870631838(407)$ & $73.926978(35)$ & $4.7 \times 10^{-7}$ \\
${ }^{74} \mathrm{Rb}$ & $64.8 \mathrm{~ms}$ & $0.8708354985(886)$ & $73.9442716(75)$ & $1.0 \times 10^{-7}$ \\
${ }^{75} \mathrm{Rb}$ & $19.0 \mathrm{~s}$ & $0.882545317(248)$ & $74.938567(21)$ & $2.8 \times 10^{-7}$ \\
${ }^{76} \mathrm{Rb}$ & $36.5 \mathrm{~s}$ & $0.8942812011(183)$ & $75.9350753(16)$ & $2.1 \times 10^{-8}$ \\
${ }^{77} \mathrm{Rb}$ & $3.77 \mathrm{~min}$ & $0.9060031627(161)$ & $76.9304016(14)$ & $1.8 \times 10^{-8}$ \\
${ }^{79} \mathrm{Rb}$ & $22.9 \mathrm{~min}$ & $0.9294816589(265)$ & $78.9239899(23)$ & $2.9 \times 10^{-8}$ \\
${ }^{80} \mathrm{Rb}$ & $33.4 \mathrm{~s}$ & $0.9412413104(233)$ & $79.9225165(20)$ & $2.5 \times 10^{-8}$ \\
${ }^{83} \mathrm{Rb}$ & $86.2 \mathrm{~d}$ & $0.9764851446(293)$ & $82.9151142(25)$ & $3.0 \times 10^{-8}$ \\
${ }^{84} \mathrm{Sr}$ & stable & $0.9882421861(182)$ & $83.9134192(15)$ & $1.8 \times 10^{-8}$ \\
\hline \hline
\end{tabular}

$\overline{\bar{a} \text { An isomeric contamination cannot be excluded. The frequency ratio and mass given in the table are of the isomeric }}$ mixture.

${ }^{\mathrm{b}}$ This result has been published previously [24].

${ }^{\mathrm{c}}$ This result (mass excess only) has been published previously [13]. 

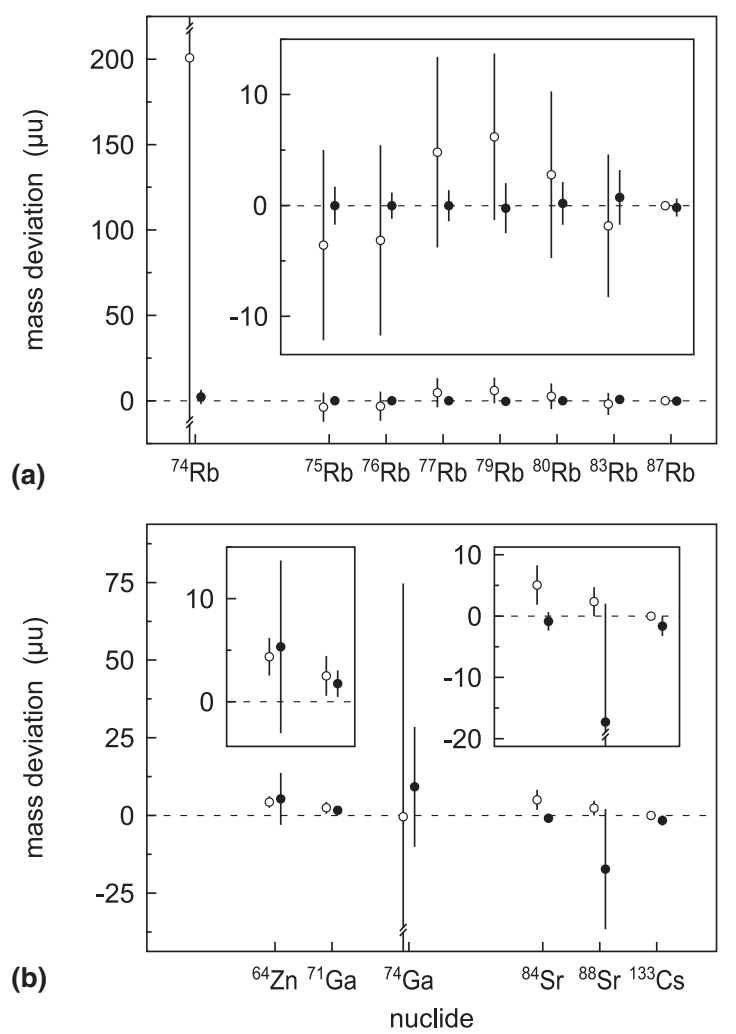

FIG. 2. Overview of the measured masses, compared with the literature values of the 1995 Atomic-Mass Evaluation [26]: (a) Rb isotopes; (b) other measured nuclides. The empty circles are the AME1995 data (except for ${ }^{87} \mathrm{Rb}$ and ${ }^{133} \mathrm{Cs}$, which are from Ref. [27]), and the filled circles are the present ISOLTRAP values; all are shown relative to the new adjusted values (dashed horizontal line, see Sec. IV). The insets are enlarged portions of the graph.

prior literature values is shown in Fig. 2. Observed deviations between our measurements and previous data are discussed in detail in the following section.

\section{ATOMIC-MASS EVALUATION}

The mass excess $D$ of a nuclide is defined by the relation

$$
D=m-A \mathrm{u},
$$

where $A$ is the atomic mass number of the nuclide and $m$ is its atomic mass. The mass excesses of all known nuclides are tabulated in the Atomic-Mass Evaluation (AME), which is constantly updated and published every few years. The most recent published version dates from 2003 [25]. Because a few of the masses reported here were already included in the AME2003, all comparisons with literature values will be made relative to the AME1995 [28].

The AME is the result of a least-squares adjustment of the most precise available experimental data on mass measurements and decay and reaction energies. Only in a very small minority of mass measurements does the measured quantity connect the mass of the studied nuclide directly to ${ }^{12} \mathrm{C}$, the microscopic mass standard. In these so-called absolute mass measurements, the mass of the reference ion is exactly known and does not contribute to the uncertainty of the final result. In most cases, however, a measurement supplies a value for the mass difference between any two nuclides; sometimes more than two nuclides are involved, for instance when the calibration is explicitly treated as part of the measured quantity. For the purpose of the AME, the frequency ratios of the ISOLTRAP mass results must be expressed as linear equations. The exact procedure for this transformation is described in detail in Ref. [29].

The ensemble of these connections spans a web of mass relations over the entire chart of the nuclides; mathematically, they represent an overdetermined set of linear equations. Each nuclide can be linked to several other nuclides. Before the actual mass adjustment is carried out, some similar measurements among the same nuclides are pre-averaged. Some others, which have been superseded by measurements with a much smaller uncertainty, are labeled as "weight much less than that of a combination of other data." These equations are not used for the following step of the evaluation. As the central step in the AME, a least-squares analysis is carried out on the remaining several hundred linear equations for all nuclides having more than one link to other nuclides (primary nuclides). The adjusted masses of those are subsequently used to determine the nuclides with only one link to another nuclide (secondary nuclides).

\section{A. Treatment of the input values}

Among the prior mass data discussed in this section, two sets of measurements stand out because, like our present data, they span a wide range of rubidium isotopes: These are the data obtained with a magnetic-sector spectrometer and early ISOLTRAP measurements, both gathered at the ISOLDE facility at the synchrocyclotron [29,30]. In the work of Epherre et al., $600-\mathrm{MeV}$ protons were used to produce neutron-deficient rubidium isotopes $\left({ }^{74-84} \mathrm{Rb}\right)$ through spallation from a niobium target and neutron-rich isotopes $\left({ }^{85-99} \mathrm{Rb}\right)$ through fragmentation from a uranium target. The mass-separated $60-\mathrm{keV}$ ions from ISOLDE were implanted on a tantalum tube, then reionized and accelerated to $9 \mathrm{keV}$. The commercial Mattauch-Herzog-type spectrometer used in these measurements consisted of a $45^{\circ}$ electrostatic deflector, an $80^{\circ}$ dipole magnet, an electron multiplier detector, and narrow entrance and exit slits. In such a device, the electric potentials that are applied for the acceleration and deflection must be kept strictly proportional and are varied to allow ions of different mass to traverse the entire apparatus. The relative masses of two particles are then inversely proportional to the electric potentials required for transmission.

To minimize systematic effects, each nuclide of interest was measured relative to two better-known isotopes, though mathematically speaking the relations between these mass triplets are perfectly symmetric. The sets of mass relations were later brought into a format compatible with the AME and a local mass adjustment was performed by the original authors [31]. As these 60 mass triplets (among which 22 involving the rubidium isotopes reported in this work) were incorporated into the AME1983 [32], it was found that the 
partial consistency factor of the ensemble was well above 1, and all uncertainties were thus multiplied by a discrete factor 1.5 to make up for possible undetected systematic effects. That factor was later raised to 2.5 in the course of the adjustment for the AME1995 [28].

In the work of Otto et al., the isotopes ${ }^{75-87} \mathrm{Rb}$ (along with a chain of strontium isotopes) were measured in four separate data-taking periods [29]. At the time, ISOLTRAP was not yet equipped with the RFQ buncher, and the stopping of $60-\mathrm{keV}$ ions from ISOLDE was achieved by implanting them on a rotatable rhenium foil. Because of the added delay in rotating and heating the foil, the short-lived nuclide ${ }^{74} \mathrm{Rb}$ was not accessible in that experiment. The overall lower efficiency compared with the current setup led to higher statistical uncertainties than in the present work. Furthermore, prior to the carbon cluster studies of Ref. [20], a relative systematic uncertainty of $10^{-7}$ was added in quadrature to all frequency ratios [33], thereby limiting the attainable overall relative precision to that value.

Nevertheless, that work improved the mass uncertainty of the entire isotopic chain of rubidium and determined all unstable isotopes except for ${ }^{84,86} \mathrm{Rb}$ to at least $60 \%$. With uncertainties improved by several orders of magnitude as compared to the measurements of Ref. [31], the existence of a systematic effect in the data of Audi et al., which was indirectly suggested by the mass adjustment in 1983, was independently confirmed. As will be shown in detail in the following, the data of Otto et al. [29] are in excellent agreement with our present results, and the need for a coverage factor for the data of Ref. [31] is retained.

The results of these two sets of measurements, as well as all other prior data that had an influence on the respective masses in the AME1995, are shown alongside our results in Figs. 3(a) and 3(b) for all nuclides except ${ }^{87} \mathrm{Rb}$ and ${ }^{133} \mathrm{Cs}$. The mass excesses for each measurement were calculated by using the reference masses obtained from the present midstream adjustment. All masses are plotted relative to the new adjusted masses, including our data (see Sec. IV B).

The reference codes used in these figures are the $\mathrm{Nu}$ clear Science Reference (NSR) keynumbers maintained by the National Nuclear Data Center in Brookhaven [34]. A correspondence with the bibliographical references of this article is given in Table II. In the following, a comparison of our results with all prior data that had an influence on the masses in the AME1995 is discussed in detail in order of mass number $A$, then proton number $Z$.

\section{1. ${ }^{64} \mathrm{Zn}$}

The mass of the stable ${ }^{64} \mathrm{Zn}$ was previously determined mainly by a measurement of the negatron energy spectrum in the radioactive decay of ${ }^{64} \mathrm{Cu}\left(T_{1 / 2}=12.7 \mathrm{~h}\right)(1983 \mathrm{Ch} 47$ [47]). Radioactive copper was obtained by irradiating a high-purity metallic $\mathrm{Cu}$ sample of natural isotopic composition at a nuclear reactor, and the $\beta$ spectrum was then recorded with the doublefocusing spectrometer at the National Physical Laboratory in Teddington (UK). The spectrometer was calibrated with $\mathrm{K}$ conversion electrons in ${ }^{137} \mathrm{Ba}$ from a ${ }^{137} \mathrm{Cs}$ source. The $\beta$
TABLE II. Reference key relating the alphabetical reference codes used in Figs. 3(a) and 3(b) (Nuclear Science Reference keynumbers) to the bibliographical references. The references not taken from the NSR file are followed by a dagger; these are not available in NSR at present.

\begin{tabular}{|c|c|c|c|}
\hline $\begin{array}{l}\text { NSR } \\
\text { keynumber }\end{array}$ & $\begin{array}{l}\text { Bibliographical } \\
\text { reference }\end{array}$ & $\begin{array}{c}\text { NSR } \\
\text { keynumber }\end{array}$ & $\begin{array}{l}\text { Bibliographical } \\
\text { reference }\end{array}$ \\
\hline 1962Ei02 & [35] & $1984 \mathrm{Ha} . \mathrm{A}^{\dagger}$ & [48] \\
\hline 1963Ri07 & [36] & 1987 St11 & [49] \\
\hline 1971Bo01 & [37] & 1987Vi01 & [50] \\
\hline 1971Mo02 & [38] & 1987Wi15 & [51] \\
\hline 1971Ot01 & [39] & 1989Wi05 & [52] \\
\hline 1972Er05 & [40] & 1993Di03 & [53] \\
\hline 1972Ja.A ${ }^{\dagger}$ & [41] & 1994Ot01 & [29] \\
\hline 1973Ва56 & [42] & 1995Le19 & [54] \\
\hline 1975Be. $\mathrm{B}^{\dagger}$ & [43] & 2003Fi.A ${ }^{\dagger}$ & [55] \\
\hline 1975De. $\mathrm{A}^{\dagger}$ & [44] & $2007 \mathrm{Gu} 09$ & [22] \\
\hline 1975We24 & [45] & 2003Pi08 & [56] \\
\hline 1976Jo01 & [46] & $2005 \mathrm{Si} 34$ & [57] \\
\hline 1982Au01 & [31] & 2006Lu. $1^{\dagger}$ & [58] \\
\hline $1983 \mathrm{Ch} 47$ & [47] & & \\
\hline
\end{tabular}

endpoint was extracted with standard Fermi-Kurie analysis. The connection has since become primary owing to other data involving ${ }^{64} \mathrm{Cu}$ and is in moderate disagreement with these (1.6 $\sigma$ deviation).

To a lesser degree, the mass was influenced by $\gamma$ spectroscopy measurements after neutron capture into ${ }^{64} \mathrm{Zn}$, performed separately at Forschungszentrum Karlsruhe (Germany) (1971Ot01 [39]) and Reactor Centrum Nederland in Petten (1975De.A [44]). Mass-spectrometry data obtained at Notre Dame University, Indiana, using the ${ }^{64} \mathrm{Zn}(d, t)^{63} \mathrm{Zn}$ reaction (1976Jo01 [46]), also made a minor contribution to the mass. Detailed studies of the $\gamma$ spectrum of ${ }^{65} \mathrm{Ga}$ produced through proton bombardment of stable ${ }^{64} \mathrm{Zn}$ were performed at the University of Helsinki in 1975 (1975We24 [45]) and again in 1987 (1987Vi01 [50]). The $Q$ values deduced from these had no influence on the mass of ${ }^{64} \mathrm{Zn}$ in the AME1995, but they do contribute to $D\left({ }^{64} \mathrm{Zn}\right)$ in the current adjustment owing to a much improved ${ }^{65} \mathrm{Ga}$ mass [22]. As shown in the figure, with the exception of Ref. [47], all of these measurements agree well with each other. The apparent deviation of the AME1995 mass excess is due to a change of almost $4 \mathrm{keV}$ in the mass excess of ${ }^{64} \mathrm{Cu}$ between 1995 and today.

A more recent $(n, \gamma)$ datum was obtained in the framework of a coordinated research project of the International Atomic Energy Agency (IAEA) with a view to improving data required for prompt $\gamma$-ray activation analysis (2003Fi.A [55]). The adopted neutron separation energies in that publication result from neutron capture experiments performed at several participating institutes, with the vast majority stemming from spectroscopic measurements carried out at the Budapest Research Reactor (Hungary). In that setup, the calibration of the nonlinear $\gamma$-ray energy function was performed with a sophisticated procedure that is intended to overcome possible long-term instabilities. Nevertheless, in the course of incorporating the data of Ref. [55] into the AME2003, it 

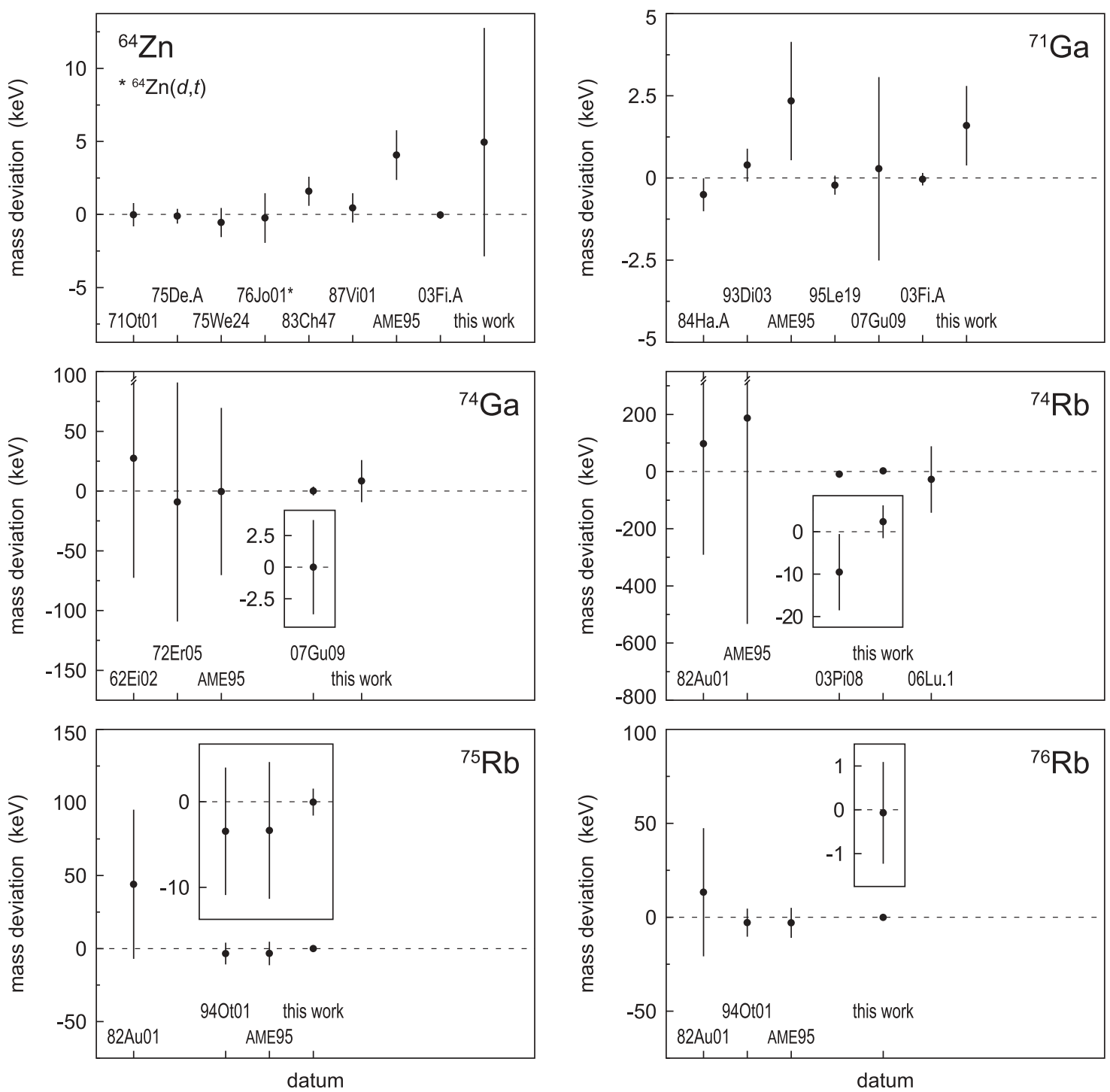

FIG. 3. (a) Comparison of the mass excesses of ${ }^{64} \mathrm{Zn},{ }^{71,74} \mathrm{Ga}$, and ${ }^{74-76} \mathrm{Rb}$ from this work with selected prior measurements; all are shown relative to the new adjusted values (dashed horizontal line; see Sec. IV B). The insets are enlarged portions of the graph. Masses were calculated by using the most recent adjusted masses of reference nuclides or reaction partners. A correspondence of the indicated keynumbers (where the first two digits of the year have been omitted) to bibliographical references is given in Table II.

became apparent that not all systematic effects had been properly accounted for, and an additional relative uncertainty of $2 \times 10^{-5}$ was added in quadrature to all results (see the comment on p. 186 of Ref. [25]). Independently from that added uncertainty, the primary result of that measurement agrees with the prior $(n, \gamma)$ data.

Our result has a fivefold larger uncertainty than the AME1995 value and is in good agreement with the previous measurements. Because of the better precision of other data, in particular the $(n, \gamma)$ measurements, our result is outweighed and is included in the AME merely as a consistency check.

\section{2. ${ }^{71} \mathrm{Ga}$}

The AME1995 mass excess for the stable nuclide ${ }^{71} \mathrm{Ga}$ was determined in equal parts by two measurements of the $Q$ value of the decay ${ }^{71} \mathrm{Ge}(\epsilon)^{71} \mathrm{Ga}$ (1984Ha.A [48],
1993Di03 [53]), which proceeds without the emission of $\gamma$ radiation. In both these experiments, pure ${ }^{71} \mathrm{Ge}\left(T_{1 / 2}=\right.$ $11.2 \mathrm{~d}$ ) was obtained by irradiating samples of stable germanium with neutrons at nuclear reactors and allowing shorterlived contaminants to decay away. The internal bremsstrahlung spectrum was recorded with Ge and/or GeLi semiconductor detectors and the decay energies were extracted from the spectra by comparison with theoretical predictions. In the publication of DiGregorio et al., the uncertainty of the $Q$ value is stated as being composed of a statistical component of $0.1 \mathrm{keV}$ and a relative systematic component of $0.1 \%$. In light of the non-negligible disagreement with the result of Ref. [48], a slightly underestimated systematic uncertainty was surmised-in particular concerning the calibration of the detector response- and the value was entered into the AME with a total uncertainty of $0.5 \mathrm{keV}$. A slightly more recent study using the same technique (1995Le19 [54]) was in good 

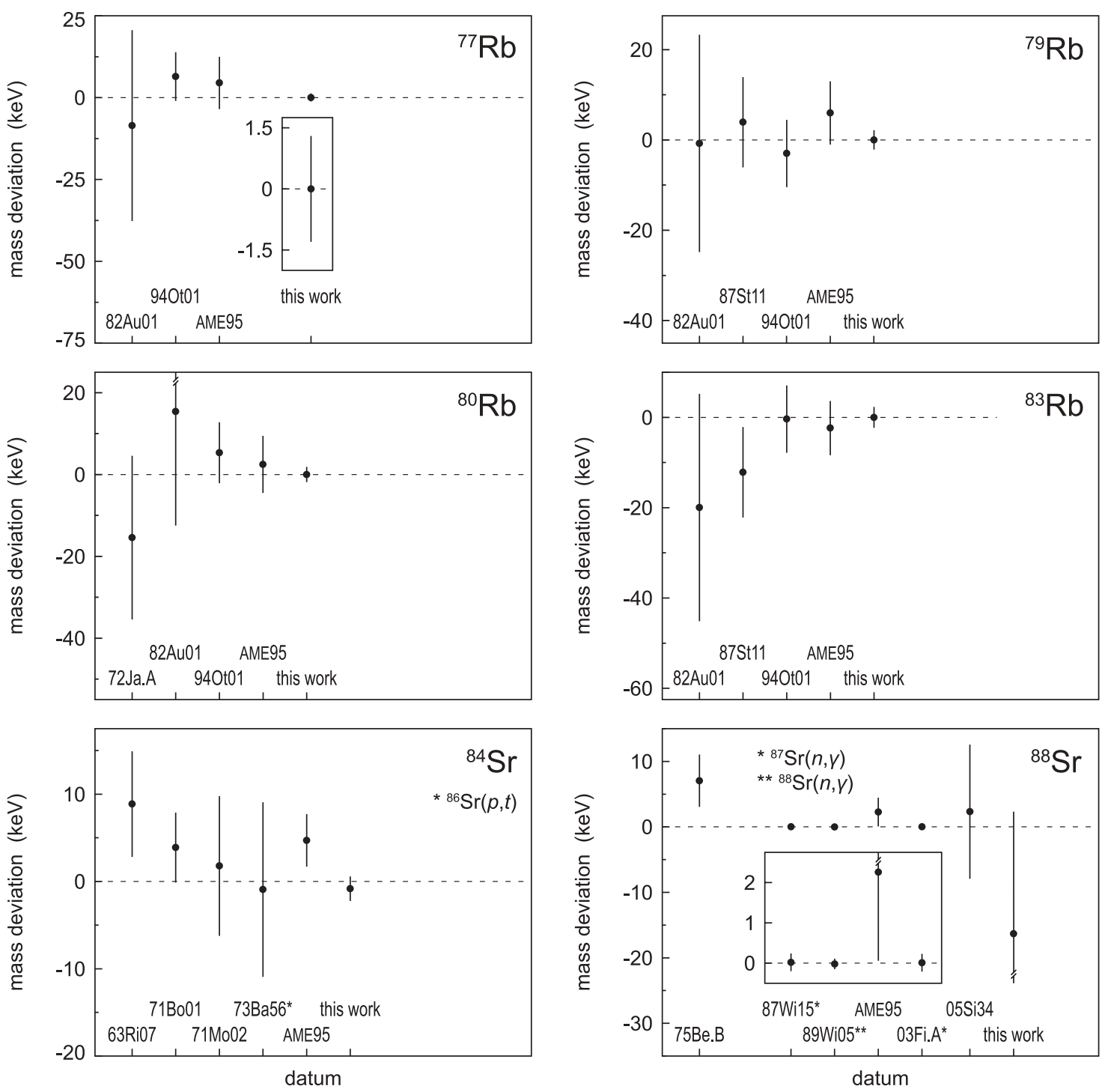

FIG. 3. (b) Continuation of Fig. 3(a) for the nuclides ${ }^{77,79,80,83} \mathrm{Rb}$ and ${ }^{84,88} \mathrm{Sr}$.

agreement with the prior results. Again, the apparent deviation of the AME1995 value is due to a change in the mass of the reaction partner in the previous measurements.

Further mass values also obtained since the compilation of the AME1995 stem from another ISOLTRAP data-taking period, dedicated to nickel, copper, and gallium nuclides around $N=40$ (2007Gu09 [22]), and from $(n, \gamma)$ data collected within the aforementioned IAEA project [55]. Our present datum agrees with the other results to within $1.3 \sigma$. Since the aforementioned prior data carry comparable or smaller uncertainties, they are all retained and contribute to the new mass value.

\section{3. ${ }^{74} \mathrm{Ga}$}

The nuclide ${ }^{74} \mathrm{Ga}$ is believed to have an isomeric state with a half-life of $9.5 \mathrm{~s}$ and an excitation energy of $59.6 \mathrm{keV}$ [59]. However, $\mathrm{Nb}$ foil targets are known to exhibit a slow release (minutes) for the element gallium. Furthermore, measurements of the yield of ${ }^{74} \mathrm{Ga}$ have shown no evidence for the isomeric state whatsoever [60]. It is therefore inferred that the ISOLDE yield of the isomer is no larger than $10 \%$ that of the ground state.

When both the ground state and an excited nuclear state of a nuclide are populated, the experimentally measured mass excess $D_{\exp }^{*}$ is shifted from the ground-state value $D_{\exp }[31]$ :

$$
D_{\exp }^{*}=D_{\exp }+\frac{R}{R+1} \Delta E,
$$

where $R=Y_{m} / Y_{g}$ is the ratio of the yield of the nuclide in the isomeric state to that in the ground state and $\Delta E$ is the excitation energy of the isomeric state. The measured mass excess must therefore be corrected by the quantity $-R /(R+$ 1) $\times \Delta E$. Based on the previously stated yield estimate, the ratio $R$ is presumed to follow a uniform probability distribution between 0 and 0.1 in the case of ${ }^{74} \mathrm{Ga}$, corresponding to a central value of 0.05 and a standard uncertainty of $0.05 / \sqrt{3}$. The correction for the mass excess then becomes 
$-2.8(1.6) \mathrm{keV}$, and the corrected mass excess is $D=$ $-68041(18) \mathrm{keV}$.

The AME1995 mass of ${ }^{74} \mathrm{Ga}$ was determined in equal parts by $\beta$ endpoint measurements of the two $\beta^{-}$decays that link the nuclide to the decay chain from ${ }^{74} \mathrm{Co}$ to the stable ${ }^{74} \mathrm{Ge}$ (1962Ei02 [35], 1972Er05 [40]). In the experiment of Eichler et al., ${ }^{74} \mathrm{Ga}$ was produced from the ${ }^{74} \mathrm{Ge}(n, p){ }^{74} \mathrm{Ga}$ reaction. The negatron spectrum of the ${ }^{74} \mathrm{Ga}\left(\beta^{-}\right)^{74} \mathrm{Ge}$ decay was first recorded without a coincidence condition and two groups with $\beta$ endpoint energies of 2450(100) and $3800(300) \mathrm{keV}$ were found. Using a coincidence with 2350-keV $\gamma$ rays, the lower-energy group was then identified as populating the $2950-\mathrm{keV}$ level in ${ }^{74} \mathrm{Ge}$, yielding a decay energy of $Q_{\beta^{-}}=5400(100) \mathrm{keV}$.

Erdal et al. produced ${ }^{74} \mathrm{Zn}$ from the ${ }^{76} \mathrm{Ge}(p, 3 p)^{74} \mathrm{Zn}$ reaction by bombarding a molten germanium target with $600-\mathrm{MeV}$ protons and recorded the negatron spectrum from the ${ }^{74} \mathrm{Zn}\left(\beta^{-}\right)^{74} \mathrm{Ga}$ decay in coincidence with $\gamma$ rays at 70 $200 \mathrm{keV}$. From this, the $\beta$ endpoint energy was found to be $E_{0}=2100(100) \mathrm{keV}$. By using the additional information that the $\beta$ decay populates the $251.8-\mathrm{keV}$ level in the daughter nucleus, a total decay energy of $Q_{\beta^{-}}=2350(100) \mathrm{keV}$ was deduced and entered into the AME.

The earlier ISOLTRAP datum of Ref. [22] completely determines the mass of ${ }^{74} \mathrm{Ga}$. All prior data are outweighed. The present datum is in excellent agreement with all previous results. Since it is 4.5 times less precise than the earlier ISOLTRAP value, it is also outweighed.

\section{4. ${ }^{74} \mathrm{Rb}$}

Prior to the AME1995, the mass of the very short-lived ${ }^{74} \mathrm{Rb}$ had been measured only once, in the work of Audi et al. already mentioned (1982Au01 [31]). In fact, two separate triplet measurements $\left({ }^{75,76,74} \mathrm{Rb}\right.$ and ${ }^{75,77,74} \mathrm{Rb}$ ), which agree well with each other, contributed to the ${ }^{74} \mathrm{Rb}$ datum.

As was outlined above, the $F t$ value for a superallowed decay is obtained from the experimental quantities' half-life $T_{1 / 2}$, branching ratio $R$, and decay energy $Q$, along with the theoretical corrections. Conversely, any one of $T_{1 / 2}, R$, and $Q$ can be inferred from the other two and the mean $F t$ value for all other superallowed decays. Again, the corrections are required as well. In this way, a new measurement of the branching ratio of the superallowed branch of ${ }^{74} \mathrm{Rb}\left(\beta^{+}\right)^{74} \mathrm{Kr}$ was used to infer the $Q$ value (2003Pi08 [56]), which Piechaczek et al. determined as $Q=10405(9) \mathrm{keV}$.

The new ISOLTRAP mass is in very good agreement with the combined value of the two triplets from Ref. [31], but it improves the uncertainty by more than two orders of magnitude as compared with the AME1995. A measurement also made at ISOLDE in 2000 with the transmission mass spectrometer MISTRAL (2006Lu.1 [58]) yielded a mass excess of $-51940(120) \mathrm{keV}$, confirming our mass using a different measurement principle. Because of the much higher precision of our datum, the other two experimental results are outweighed, while the prediction of Ref. [56] is retained and continues to make a contribution to the mass excess.

$$
\text { 5. }{ }^{75-77} \mathbf{R b}
$$

The former ISOLTRAP measurements (1994Ot01 [29]) with a relative uncertainty of about $10^{-7}$ completely determined the mass excesses of ${ }^{75} \mathrm{Rb},{ }^{76} \mathrm{Rb}$, and ${ }^{77} \mathrm{Rb}$. The older triplet data of Audi et al. are in good agreement with the Penning trap values once the confidence factor has been applied to the uncertainties. The slightly deviating AME1995 values are due to changes in the mass of ${ }^{85} \mathrm{Rb}$ in the meantime. Our present measurements reproduce the prior ISOLTRAP data well and reduce their uncertainties by factors ranging from 5 to 7 . Because of the higher precisions of the new results, the old values are outweighed.

\section{6. ${ }^{79} \mathbf{R b}$}

In the case of ${ }^{79} \mathrm{Rb}$, the mass spectrometry data of Audi et al. and the prior ISOLTRAP datum did not exclusively determine the AME1995 mass. A significant contribution of about onethird to the mass excess of ${ }^{79} \mathrm{Rb}$ was due to measurements on the ${ }^{78} \mathrm{Kr}\left({ }^{3} \mathrm{He}, d\right){ }^{79} \mathrm{Rb}$ reaction performed at the University of Pennsylvania (1987St11 [49]). In the work of Stephans et al., a gas cell target was filled with $99.5 \%$-pure ${ }^{78} \mathrm{Kr}$ and bombarded with an $18-\mathrm{MeV}^{3} \mathrm{He}$ beam. The outgoing deuterons were momentum-analyzed in a magnetic spectrograph and detected on photographic emulsion plates. The $Q$ value for the reaction was extracted from the spectrum by a comparison with the spectra for other krypton isotopes for which the reaction energy was well known.

The ${ }^{79} \mathrm{Rb}$ mass derived from this datum, using the AME1995 value for the mass excess of ${ }^{78} \mathrm{Kr}$, disagreed strongly with the mass triplet value, but also with the ISOLTRAP results of Ref. [29]. However, this deviation was entirely due to a recent change of more than $-20 \mathrm{keV}$ in the mass of ${ }^{78} \mathrm{Kr}$, which was triggered by new data from an ISOLTRAP measurement campaign dedicated to neutron-deficient krypton isotopes [10]. In that work, the strong deviation of the ISOLTRAP result from the prior literature value was in part explained by a mass doublet value from Ref. [36] that had been incorrectly transcribed into the AME1964 [61].

The present result agrees well with the previous data, including the mass excess derived from the $Q$ value of Stephans et al. if the corrected mass excess for ${ }^{78} \mathrm{Kr}$ is used. All prior data are outweighed.

\section{7. ${ }^{80} \mathbf{R b}$}

The prior ISOLTRAP measurement almost exclusively determined the mass excess of ${ }^{80} \mathrm{Rb}$ in the AME1995. A minor contribution stemmed from a study of the ${ }^{80} \operatorname{Kr}(p, n)^{80} \mathrm{Rb}$ reaction performed at Triangle Universities Nuclear Laboratory in Durham, North Carolina (1972Ja.A [41]). A gas cell containing krypton gas enriched in neutron-deficient isotopes was placed in the proton beam of a tandem van de Graaff accelerator. Since this $(p, n)$ reaction is endothermic, the (negative) $Q$ value is equal to the proton energy required for the reaction to set in. To determine the threshold, Jaffe et al. recorded positron events from the decay of the reaction product ${ }^{80} \mathrm{Rb}$. A selection of positron energies above the $\beta$ endpoint of ${ }^{82} \mathrm{Rb}$ suppressed background from the ${ }^{82} \operatorname{Kr}(p, n)^{82} \mathrm{Rb}$ 
reaction. From a graphical analysis of the positron yield versus the incident proton energy, the authors obtained a threshold of $6566(2) \mathrm{keV}$, which corresponds to a $Q$ value of $-6484(20) \mathrm{keV}$ once the energy loss in the tantalum entrance window to the gas cell has been taken into account.

The first Penning trap datum of Otto et al. agrees with the $Q$ value of Ref. [41] and with the mass excess deduced from the six mass triplets of Audi et al. Our present result indicates a slightly more bound nucleus than suggested by the prior ISOLTRAP value, but well within the error bars of both measurements. With an uncertainty of $2 \mathrm{keV}$, the new ISOLTRAP value outweighs all previous measurements.

\section{8. ${ }^{83} \mathrm{Rb}$}

The situation of ${ }^{83} \mathrm{Rb}$ prior to this work was similar to that of ${ }^{79} \mathrm{Rb}$. In addition to the data of Refs. [31] and [29], the result from the $\left({ }^{3} \mathrm{He}, d\right)$ reaction studies of Stephans et al. contributed about one-third to the adjusted mass excess. Here the deviation of that datum from the more recent results was less pronounced than in the case of ${ }^{79} \mathrm{Rb}$, but a slight disagreement of $1.1 \sigma$ persists even when using the most recent value for the mass of ${ }^{82} \mathrm{Kr}$ (also in part due to Ref. [10]) to calculate the mass excess.

Our new datum agrees well with the mass triplet data and the prior ISOLTRAP measurement. With an almost threefold improvement of the precision brought about by the present work, all prior data are now outweighed.

\section{9. ${ }^{84} \mathrm{Sr}$}

At the time of the AME1995, the mass excess of the stable nuclide ${ }^{84} \mathrm{Sr}$ was due to measurements employing a number of complementary techniques. The oldest datum that still carried significant weight in the AME1995 was from the 16-inch double-focusing spectrometer at the University of Minnesota (1963Ri07 [36]). In that work, Ries et al. measured the masses of 42 stable nuclides relative to hydrocarbon molecules with the same mass numbers (mass doublets). An AME adjustment incorporating these results revealed a large partial consistency factor, and the uncertainties of all mass differences were expanded by a factor 2.5. (Note that this factor is still required notwithstanding the correction of the aforementioned typographical error.)

The strongest contribution to the mass of ${ }^{84} \mathrm{Sr}$ stemmed from a study of the negatron decay of ${ }^{84} \mathrm{Rb}$ (1971Bo01 [37]), a weak decay branch $(R=3.8 \%)$ observed in addition to the main $\beta^{+}$and $\epsilon$ channels. Booij et al. produced a sample of ${ }^{84} \mathrm{Rb}$ by $\alpha$ bombardment of stable bromine. The positron and negatron spectra were measured with a double-focusing spectrometer. Whereas the $\beta^{-}$decay proceeds only to the ground state of ${ }^{84} \mathrm{Sr}$, the $\beta^{+}$decay can populate either of two excited states in addition to the ground state of ${ }^{84} \mathrm{Kr}$. The authors separately measured the endpoints for the decay to the ground and the first excited state, as well as the level energy of the first excited state. The evaluators of the AME later noted an inconsistency between the difference of the two $\beta^{+}$endpoint energies and the accepted level energy from spectroscopic measurements, and quadratically added an uncertainty of $3 \mathrm{keV}$ to all reported endpoint energies. This also affected the
${ }^{84} \mathrm{Rb}\left(\beta^{-}\right){ }^{84} \mathrm{Sr}$ result, whose overall uncertainty was increased to $4 \mathrm{keV}$.

Further mass data were taken from reaction $Q$ values obtained by researchers at the University of Oxford (UK) (1971Mo02 [38]) and Oak Ridge National Laboratory, Tennessee (1873Ba56 [42]). All of the mentioned data agree moderately well with each other (within the increased uncertainties), with a maximal deviation of $1.5 \sigma$ in the case of the mass doublet value. Our present result supplies by far the most precise datum for the ${ }^{84} \mathrm{Sr}$ mass. It is in agreement with all prior data except that of Ref. [36], whose deviation remains despite the applied coverage factor. For the new mass adjustment, all prior data discussed so far are retained, except for that of Ref. [42], but their contribution to the new mass is significantly reduced owing to the higher precision of our result.

\section{0. ${ }^{88} \mathrm{Sr}$}

The AME1995 mass excess of the stable ${ }^{88} \mathrm{Sr}$ was essentially derived from two separate measurements of the $Q$ value of the ${ }^{87} \mathrm{Sr}(n, \gamma){ }^{88} \mathrm{Sr}$ and the ${ }^{88} \mathrm{Sr}(n, \gamma){ }^{89} \mathrm{Sr}$ reactions performed within a period of three years at the ILL high-flux reactor in Grenoble (France) (1987Wi15 [51], 1989Wi05 [52]). An older datum from ${ }^{88} \mathrm{Sr}(p, \gamma){ }^{89} \mathrm{Y}$ reaction energy studies (1975Be.B [43]) deviated strongly from these but was retained in the AME1995 because the two sets of $(n, \gamma)$ data were from the same group and might thus have been prone to a common undetected systematic effect. With the inclusion of additional $(n, \gamma)$ data [55], the measurement of Bertsche et al. was marked "well-documented data, or data from regular reviewed journals, which disagree with other well-documented values" and excluded from the adjustment.

The present ISOLTRAP value for the mass excess of ${ }^{88} \mathrm{Sr}$ stems from a single TOF cyclotron resonance recorded during the data-taking period in 2000. This accounts for the rather large uncertainty of our datum. It is in agreement with a prior ISOLTRAP datum (2005Si34 [57]) and deviates by $0.9 \sigma$ from the more precise spectroscopic data. Since the uncertainty of the present mass excess is ten times larger than the combined previous results, it is outweighed.

\section{1. ${ }^{87} \mathrm{Rb}$ and ${ }^{133} \mathrm{Cs}$}

The MIT Penning trap mass measurements of the stable nuclides ${ }^{85,87} \mathrm{Rb}$ and ${ }^{133} \mathrm{Cs}$ reached relative uncertainties better than $2 \times 10^{-10}$ [27]. A determination of the cyclotron frequency ratio of the nuclides ${ }^{87} \mathrm{Rb}$ and ${ }^{133} \mathrm{Cs}$ by ISOLTRAP can therefore not contribute to the final result of the AME. Our results are outweighed and are included in the AME merely for consistency checks. Our ${ }^{87} \mathrm{Rb}$ result is in perfect agreement with the MIT value, but the datum for ${ }^{133} \mathrm{Cs}$, whose mass is almost twice as large as that of all other studied nuclides, deviates from the MIT result by just over one standard deviation.

\section{B. Result of the mass adjustment}

The result of a midstream atomic-mass evaluation carried out in the course of this work is given in Table III. In columns 


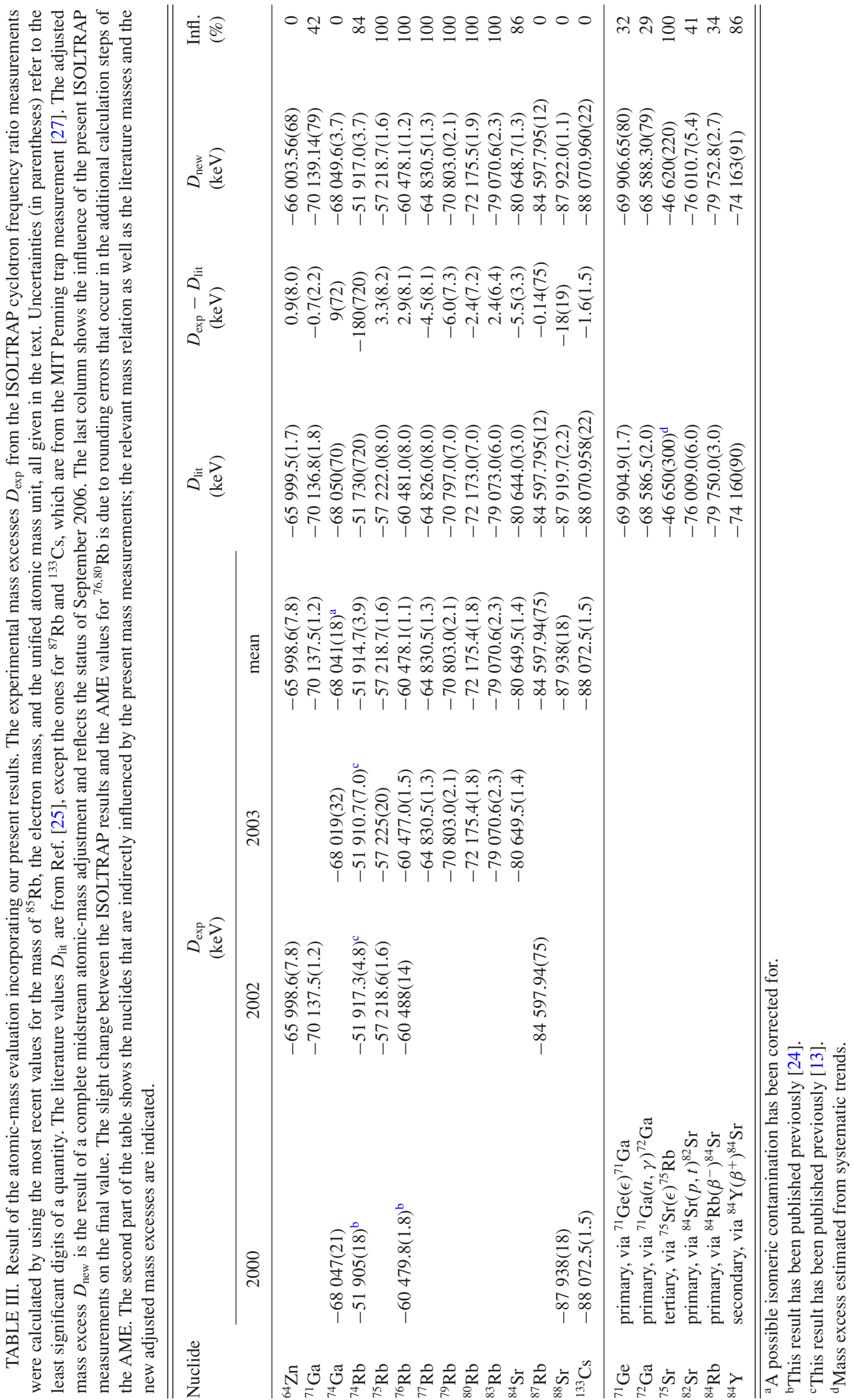


$2-5$, the mass excesses obtained during the data-taking periods 2000-2003 as well as their weighted means are presented. The sixth column gives the previous literature value [26] and the seventh column the difference between the two. The eighth column shows the new mass excess after the mass evaluation, including the present ISOLTRAP results as well as all other mass data that have become available since the AME1995. It reflects the status of September 2006. The influence of the ISOLTRAP data on the final mass excesses is given in the last column. For the nuclide ${ }^{74} \mathrm{Ga}$, the correction from a possible presence of an isomer has been applied.

\section{1. ${ }^{71,74} \mathrm{Ga}$}

Despite the fact that our present measurement on ${ }^{71} \mathrm{Ga}$ is not the most precise of all available data, it makes the largest contribution to the mass (42\%). This is due to the excellent knowledge of the reference nuclide ${ }^{85} \mathrm{Rb}$ as compared with the daughter nuclide ${ }^{72} \mathrm{Ga}$ produced in the spectroscopic studies [55] and the decay parent ${ }^{71} \mathrm{Ge}$ from the endpoint measurements [48,53]. These data contribute $31 \%$ and $18 \%$, respectively, to the new mass.

As was already mentioned, the most recent ISOLTRAP measurements on ${ }^{74} \mathrm{Ga}$, reported in Ref. [22], determine the mass to $100 \%$. The nuclide has thus become secondary.

\section{2. ${ }^{74} \mathrm{Rb}$}

Our measurements on the shortest-lived nuclide studied in the present work supply by far the most precise experimental mass value. They determine the ${ }^{74} \mathrm{Rb}$ mass to $84 \%$, with a minor contribution of $16 \%$ from the prediction assuming CVC [56]. The apparent deviation of Ref. [56] is in part repaired by a change in the mass of ${ }^{74} \mathrm{Kr}$ brought about by the recent ISOLTRAP measurement on krypton isotopes already mentioned [10]. The remaining disagreement of $1.1 \sigma$ from the new value is most likely due to an incomplete knowledge of the theoretical corrections required to predict the $Q$ value of the superallowed decay from the other experimental observables. For a more detailed discussion, see Ref. [13].

\section{3. ${ }^{75-77,79,80,83} \mathbf{R b}$}

The new masses of the six rubidium isotopes ${ }^{75} \mathrm{Rb},{ }^{76} \mathrm{Rb}$, ${ }^{77} \mathrm{Rb},{ }^{79} \mathrm{Rb},{ }^{80} \mathrm{Rb}$, and ${ }^{83} \mathrm{Rb}$ are completely determined by our present measurements. With the exception of ${ }^{83} \mathrm{Rb}$, they have all become secondary nuclides in the new AME as no other experimental data with comparable precision are available. ${ }^{83} \mathrm{Rb}$ was artificially retained as a primary nuclide to allow the mass relations with ${ }^{83} \mathrm{Sr}$ to be properly taken into account.

The prior measurements that influenced the masses of these nuclides in the AME1995 are generally in good agreement with the new adjusted values. The large deviation of the datum of Stephans et al. in the case of ${ }^{79} \mathrm{Rb}$ was removed by the new ISOLTRAP datum for ${ }^{78} \mathrm{Kr}$ [10], whereas a $1.2 \sigma$ disagreement of their work with the new adjusted value of ${ }^{83} \mathrm{Rb}$ remains.

$$
\text { 4. }{ }^{84} \mathrm{Sr}
$$

A total of six primary relations between ${ }^{84} \mathrm{Sr}$ and its reaction or decay partners are retained in the present midstream mass adjustment. Out of these, the ISOLTRAP datum makes the strongest contribution to the mass, which it determines to $86 \%$. The other relations all contribute less than $6 \%$, with the flow of information mainly directed toward the partner nuclides. As we will see in the following, secondary links extend the influence of our measurement to nuclides that have not been measured directly in the present work.

\section{5. ${ }^{64} \mathrm{Zn},{ }^{87} \mathrm{Rb},{ }^{88} \mathrm{Sr}$, and ${ }^{133} \mathrm{Cs}$}

The mass excesses for these nuclides remain unaffected by the ISOLTRAP measurements presented here. Our results are in acceptable $(1.1 \sigma)$ to excellent $(0.2 \sigma)$ agreement with the adjusted masses, thereby confirming the validity of the results for the exotic nuclides.

\section{Influences on other nuclides}

The studied nuclides occupy a limited region of the nuclear chart and none of them are used as mass references (except among each other, in measurements that have largely been outweighed by the present work). Because of our excellent knowledge of the reference nuclide ${ }^{85} \mathrm{Rb}$, the flow of information is exclusively directed toward the masses of the exotic nuclides. Furthermore, the $\beta^{+}$decay products of most of the rubidium isotopes have recently been measured directly [10] and are no longer determined by the parent masses via the decay energies. Therefore, the measurements presented here have had no dramatic effect on the adjusted mass excesses of a large number of other nuclides. Nevertheless, some other nuclei are influenced by changes in the masses of their reaction or decay partners.

Among the primary nuclides, ${ }^{71} \mathrm{Ge},{ }^{72} \mathrm{Ga},{ }^{82} \mathrm{Sr}$, and ${ }^{84} \mathrm{Rb}$ receive non-negligible indirect influences from the measured nuclides. According to the new adjustment, the mass of ${ }^{71} \mathrm{Ge}$ is influenced to $32 \%$ by our measurement of ${ }^{71} \mathrm{Ga}$ via the ${ }^{71} \mathrm{Ge}(\epsilon)^{71} \mathrm{Ga}$ decay. That same nuclide also influences the mass of ${ }^{72} \mathrm{Ga}$ to $29 \%$ through spectroscopic data on the ${ }^{71} \mathrm{Ga}(n, \gamma){ }^{72} \mathrm{Ga}$ reaction. The nuclide ${ }^{82} \mathrm{Sr}$ is influenced to $41 \%$ by our data via the reaction ${ }^{84} \operatorname{Sr}(p, t){ }^{82} \mathrm{Sr}$. An analysis of the result of the mass adjustment reveals that, in addition to the directly measured rubidium isotopes, the mass of ${ }^{84} \mathrm{Rb}$ also benefits from the present results and receives a 34\% influence via the negatron decay of ${ }^{84} \mathrm{Sr}$.

Furthermore, two other nuclides are influenced through secondary connections. That is the case of ${ }^{75} \mathrm{Sr}$, which is connected to ${ }^{75} \mathrm{Rb}$ via the ${ }^{75} \mathrm{Sr}(\epsilon)^{75} \mathrm{Rb}$ electron capture decay and is determined to $100 \%$ by the present measurements. Lastly, the secondary nuclide ${ }^{84} \mathrm{Y}$ is completely determined by our measurement through the connection to its positron decay daughter ${ }^{84} \mathrm{Sr}$. A similar $\beta^{+}$link to the isomer ${ }^{84} \mathrm{Y}^{m}$ brings no additional information, as the level energy of the 4.6-s isomer is fully determined by the $Q$ value difference obtained from $\beta$ endpoint measurements [62]. 


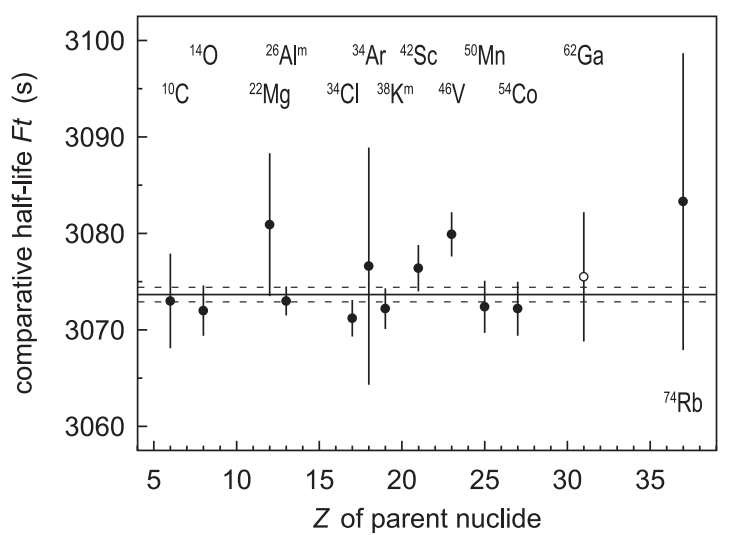

FIG. 4. Comparative half-lives $F t$ of the twelve nuclides (full circles) for which the $F t$ values are known to the highest precision (values from Ref. [8], Table IX, updated in Ref. [63]). The data point for ${ }^{62} \mathrm{Ga}$ [64], not yet included in the updated evaluation of Ref. [63], is also shown (empty circle). The current mean $F t$ value (excluding ${ }^{62} \mathrm{Ga}$ ) is represented as a solid horizontal line and its uncertainty as dashed lines.

\section{DISCUSSION AND CONCLUSION}

The mass of ${ }^{74} \mathrm{Rb}$ from the present ISOLTRAP measurement, together with the mass of ${ }^{74} \mathrm{Kr}$ from Ref. [10], yields a decay energy of $Q=10416.8(4.5) \mathrm{keV}$ and a comparative half-life of $F t=3083(15)$ s. Figure 4 shows the resulting comparative half-life alongside that of the other 12 nuclides for which the $F t$ value is known with the highest precision. The values are taken from Ref. [8], as updated in Ref. [63] (full circles). In addition, the $F t$ value of ${ }^{62} \mathrm{Ga}$ from Ref. [64] is shown in the figure (empty circle), though it has not yet been included in Hardy and Towner's evaluation and is also not used in the computation of the mean, which is represented as horizontal lines. Likewise, recent mass measurements on ${ }^{26} \mathrm{Al}^{\mathrm{m}},{ }^{38} \mathrm{Ca},{ }^{42} \mathrm{Sc}$, and ${ }^{46} \mathrm{~V}[63,65-67]$ have not yet been included in Hardy and Towner's evaluation. The $F t$ values of ${ }^{22} \mathrm{Mg},{ }^{34} \mathrm{Ar}$, and ${ }^{74} \mathrm{Rb}$ are predominantly determined by ISOLTRAP mass measurements [13,68,69].

The figure shows that in the case of ${ }^{74} \mathrm{Rb}$ the overall uncertainty of $F t$ is still somewhat larger than for the other nuclides. However, this is not due to the precision with which the decay energy is known, but rather due to the isospinsymmetry-breaking correction $\delta_{C}$ being not yet sufficiently well known and dominating the uncertainty of $F t$ [8].

The data points agree well with each other within their uncertainties and support the expectation of a constant $F t$ (reduced chi squared of a fit to a horizontal straight line $\left.\chi_{v}^{2}=1.12\right)$; they yield a mean value of $F t=3073.66(75) \mathrm{s}$ [63]. This result places a severe limit on the existence of scalar currents [70]. By using $V_{u d}$ obtained from this mean $F t$ value, along with the other two elements $V_{u s}$ and $V_{u b}$ from the most recent Particle Data Group evaluation of particlephysics data [71], the partial unitarity test of CKM yields $\left|V_{u d}\right|^{2}+\left|V_{u s}\right|^{2}+\left|V_{u b}\right|^{2}=0.9967(14)$ and thus fails by more than two standard deviations. However, as Hardy and Towner point out [70], if only the two most recent measurements of $V_{u s}[72,73]$ are considered, the unitarity of the CKM matrix is restored.

The relative uncertainties of the present mass measurements on radionuclides range from $1.8 \times 10^{-8}$ to $4.7 \times 10^{-7}$. In most cases, they are not yet limited by the residual systematic uncertainty of $8.0 \times 10^{-9}$ that was found in Ref. [20]. This means that, with better statistics, an improvement by ISOLTRAP of almost all these measurements is still possible. This is especially true for ${ }^{74} \mathrm{Rb}$, where a mass uncertainty of about $4 \mathrm{keV}$ was reached. Nevertheless, the previous AME mass value was improved by more than two orders of magnitude and our results have allowed a first meaningful determination of an $F t$ value for $A>54$. Further improvements of the mass of ${ }^{74} \mathrm{Rb}$ by as much as a factor of 5 could be achieved in future measurements, but the $F t$ value for its superallowed decay is presently limited by theoretical considerations rather than experimentally accessible quantities.

Despite the excellent agreement between virtually all $F t$ values over a wide mass range, further experiments that either target new decays or substantially improve the data on those for which prior measurements exist can still increase the stringency of the CVC test. Ultimately, they can also lead to a better control of the isospin-symmetry-breaking correction $\delta_{C}$, in particular where this correction is large, as is the case for ${ }^{62} \mathrm{Ga}$ and ${ }^{74} \mathrm{Rb}$. These heavier nuclides tend to be very short-lived and therefore represent unique challenges to trap experiments. In upcoming measurements, ISOLTRAP will be used to measure the masses of ${ }^{62} \mathrm{Ga}$ and its daughter ${ }^{62} \mathrm{Zn}$, as well as the nuclides ${ }^{26} \mathrm{Al}$ and ${ }^{14} \mathrm{O}$ on the low-mass side of the well-known superallowed emitters.

\section{ACKNOWLEDGMENTS}

The authors thank the ISOLDE Technical Group and the ISOLDE Collaboration for their assistance. This work was supported by the German Federal Ministry for Education and Research (BMBF) under Contract Nos. 06GF151, 06GF186I, 06MZ215, and 06MZ962I, by the Helmholtz Association of National Research Centers (HGF) under Contract No. VHNG-037, by the German Research Foundation (DFG) under Contract No. KE1369/1-1, by the European Commission under Contract Nos. HPRI-CT-2001-50034 (NIPNET) and HPRICT-2001-50033 (TARGISOL), and by the French IN2P3.
[1] D. Lunney, J. M. Pearson, and C. Thibault, Rev. Mod. Phys. 75, 1021 (2003).

[2] K. Blaum, Phys. Rep. 425, 1 (2006).

[3] F. Herfurth et al., J. Phys. B 36, 931 (2003).

[4] K. Blaum et al., Nucl. Phys. A752, 317 (2005).

[5] A. Kellerbauer, Int. J. Mass Spectrom. 229, 107 (2003).
[6] R. P. Feynman and M. Gell-Mann, Phys. Rev. 109, 193 (1958).

[7] W. E. Ormand, B. A. Brown, and B. R. Holstein, Phys. Rev. C 40, 2914 (1989).

[8] J. C. Hardy and I. S. Towner, Phys. Rev. C 71, 055501 (2005).

[9] I. S. Towner and J. C. Hardy, Nucl. Phys. A205, 33 (1973).

[10] D. Rodríguez et al., Nucl. Phys. A769, 1 (2006). 
[11] R. B. Cakirli, D. S. Brenner, R. F. Casten, and E. A. Millman, Phys. Rev. Lett. 94, 092501 (2005).

[12] D. S. Brenner, R. B. Cakirli, and R. F. Casten, Phys. Rev. C 73, 034315 (2006).

[13] A. Kellerbauer et al., Phys. Rev. Lett. 93, 072502 (2004).

[14] E. Kugler, Hyperfine Interact. 129, 23 (2000).

[15] M. Mukherjee et al. (to be published).

[16] G. Gräff, H. Kalinowsky, and J. Traut, Z. Phys. A 297, 35 (1980).

[17] M. König et al., Int. J. Mass Spectrom. 142, 95 (1995).

[18] L. S. Brown and G. Gabrielse, Rev. Mod. Phys. 58, 233 (1986).

[19] K. Blaum et al., J. Phys. B 36, 921 (2003).

[20] A. Kellerbauer et al., Eur. Phys. J. D 22, 53 (2003).

[21] K. Blaum et al., Phys. Rev. Lett. 91, 260801 (2003).

[22] C. Guénaut et al., Phys. Rev. C 75, 044303 (2007).

[23] G. Audi et al., Nucl. Phys. A729, 3 (2003).

[24] F. Herfurth et al., Eur. Phys. J. A 15, 17 (2002).

[25] A. H. Wapstra, G. Audi, and C. Thibault, Nucl. Phys. A729, 129 (2003); G. Audi, A. H. Wapstra, and C. Thibault, Nucl. Phys. A729, 337 (2003).

[26] G. Audi and A. H. Wapstra, Nucl. Phys. A595, 409 (1995).

[27] M. P. Bradley, J. V. Porto, S. Rainville, J. K. Thompson, and D. E. Pritchard, Phys. Rev. Lett. 83, 4510 (1999).

[28] G. Audi and A. H. Wapstra, Nucl. Phys. A595, 409 (1995).

[29] T. Otto et al., Nucl. Phys. A567, 281 (1994).

[30] M. Epherre, G. Audi, C. Thibault, R. Klapisch, G. Huber, F. Touchard, and H. Wollnik, Phys. Rev. C 19, 1504 (1979).

[31] G. Audi et al., Nucl. Phys. A378, 443 (1982).

[32] A. H. Wapstra and G. Audi, Nucl. Phys. A432, 1 (1985); A432, 55 (1985); K. Bos, G. Audi, and A. H. Wapstra, ibid. A432, 140 (1985); A. H. Wapstra, G. Audi, and R. Hoekstra, ibid. A432, 185 (1985).

[33] G. Bollen et al., Nucl. Instrum. Methods A 368, 675 (1996).

[34] National Nuclear Data Center NSR Database, http://www. nndc.bnl.gov/nsr/.

[35] E. Eichler et al., Nucl. Phys. 35, 625 (1962).

[36] R. R. Ries, R. A. Damerow, and W. H. Johnson Jr., Phys. Rev. 132, 1662 (1963).

[37] H. M. W. Booij et al., Nucl. Phys. A160, 337 (1971).

[38] J. M. Morton et al., Nucl. Phys. A161, 228 (1971).

[39] H. Ottmar et al., Nucl. Phys. A164, 69 (1971).

[40] B. R. Erdal et al., Nucl. Phys. A194, 449 (1972).

[41] A. A. Jaffe et al., in 4th International Conference on Atomic Masses and Fundamental Constants, Teddington, UK, 6-10 September 1971, edited by J. H. Sanders and A. H. Wapstra (Plenum, London, 1972), p. 236.

[42] J. B. Ball et al., Phys. Rev. C 8, 1438 (1973).
[43] U. Bertsche, F. Rauch, and K. Stelzer, in 5th International Conference on Atomic Masses and Fundamental Constants, Paris, France, 2-6 June 1975, edited by J. H. Sanders and A. H. Wapstra (Plenum, New York, 1976), p. 54.

[44] J. de Boer, in 2nd International Conference on Neutron Capture Gamma Ray Spectroscopy, Petten, Netherlands, 2-6 September 1974, edited by J. H. Sanders and A. H. Wapstra (Reactor Centrum Nederland, Petten, 1975), p. 609.

[45] Th. Weckström, I. Forsblom, and P. Holmberg, Phys. Fenn. 10, 167 (1975).

[46] P. L. Jolivette et al., Phys. Rev. C 13, 439 (1976).

[47] P. Christmas et al., Nucl. Instrum. Methods 215, 397 (1983).

[48] W. Hampel and R. Schlotz, in 7th International Conference on Atomic Masses and Fundamental Constants, DarmstadtSeeheim, Germany, 3-7 September 1984, edited by O. Klepper (GSI, Darmstadt, 1984), p. 89.

[49] G. S. F. Stephans et al., Phys. Rev. C 35, 2033 (1987).

[50] K. Vierinen, Nucl. Phys. A463, 605 (1987).

[51] Ch. Winter et al., Nucl. Phys. A473, 129 (1987).

[52] Ch. Winter et al., Nucl. Phys. A491, 395 (1989).

[53] D. E. DiGregorio, S. Gil, H. Huck, E. R. Batista, A. M. J. Ferrero, and A. O. Gattone, Phys. Rev. C 47, 2916 (1993).

[54] Y. S. Lee, M. Kobayashi, T. Hukutome, T. Horiguchi, and H. Inoue, Phys. Rev. C 51, 2770 (1995).

[55] R. B. Firestone et al., Database of Prompt Gamma Rays from Slow Neutron Capture for Elemental Analysis (IAEA, Vienna, 2006).

[56] A. Piechaczek et al., Phys. Rev. C 67, 051305(R) (2003).

[57] G. Sikler et al., Nucl. Phys. A763, 45 (2005).

[58] D. Lunney et al., Int. J. Mass Spectrom. 251, 286 (2006).

[59] A. R. Farhan, Nucl. Data Sheets 74, 529 (1995).

[60] U. Köster (private communication).

[61] J. H. E. Mattauch, W. Thiele, and A. H. Wapstra, Nucl. Phys. 67, 1 (1965); 67, 32 (1965); 67, 73 (1965).

[62] C. J. Lister, P. E. Haustein, D. E. Alburger, and J. W. Olness, Phys. Rev. C 24, 260 (1981).

[63] G. Savard et al., Phys. Rev. Lett. 95, 102501 (2005).

[64] T. Eronen et al., Phys. Lett. B636, 191 (2006).

[65] G. Bollen et al., Phys. Rev. Lett. 96, 152501 (2006).

[66] T. Eronen et al., Phys. Rev. Lett. 97, 232501 (2006).

[67] S. George et al., Phys. Rev. Lett. 98, 162501 (2007).

[68] F. Herfurth et al., Phys. Rev. Lett. 87, 142501 (2001).

[69] M. Mukherjee et al., Phys. Rev. Lett. 93, 150801 (2004).

[70] J. C. Hardy and I. S. Towner, Phys. Rev. Lett. 94, 092502 (2005).

[71] S. Eidelmann et al., Phys. Lett. B592, 1 (2004).

[72] A. Sher et al., Phys. Rev. Lett. 91, 261802 (2003).

[73] T. Alexopoulos et al., Phys. Rev. Lett. 93, 181802 (2004). 